\title{
Compilation of OCaml memory model into Power
}

1,3 E.S. Namakonov, ORCID:0000-0002-7517-9389<st070466@student.spbu.ru>

2,3,4 A.V.Podkopaev, ORCID:0000-0002-9448-6587<apodkopaev@hse.ru>

${ }^{1}$ St Petersburg University,

\section{Компиляция модели памяти OCaml в Power}

1,3 E.C. Намаконов, ORCID: 0000-0002-7517-9389<st070466@student.spbu.ru>

${ }^{2,3,4}$ A.B. Подкопаев, ORCID: 0000-0002-9448-6587<apodkopaev@hse.ru>

${ }^{1}$ Санкт-Петербургский государственный университет,

199034, Россия, Санкт-Петербург, Университетская набережная, д. 7-9

${ }_{2}^{2}$ Наииональный исследовательский университет «Высшая школа экономики ,

194100, Россия, Санкт-Петербург, Кантемировская ул., ЗА корпус

$$
{ }^{3} \text { JetBrains Research, }
$$

197342, Россия, Санкт-Петербург, Кантемировская ул., 2

${ }^{4}$ Институт им. Макса Планка: Программные Системы

67663, Германия, Кайзерслаутерн, ул. Пауль-Эрлих G26.

Аннотация. В настоящее время для языков программирования и процессоров активно разрабатываются модели памяти, направленные на решение различных проблем многопоточного ралрба расы

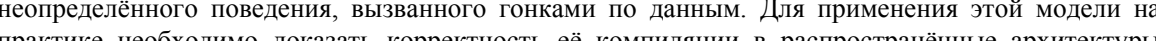
практике необхдимо доказь корректнось ее компиляции в распространённые архитектуры процессоров. На данный момент это выполнено для моделей х86 и ARM, но не для Power. Для того, ятобы упростить доказательство корректности компиляции ОCamIMM в модель Power, предлагается построить схему компиляции ОСаmIMМ в промежуточную модель памяти (IMM). Для этой модели уже доказана корректность компиляции в модель Power и другие архитектуры, поэтому доказательство корректности компиляции OCamlMM в модель Power сводится к доказательству корректности компиляции ОСamlMM в IMM. В данной работе предложена схема компиляции OCamlMM в IMM и доказана её корректность. В этой схеме используются барьеры памяти и инструкции compare-and-swap, которые позволяют исключить поведение, допустимое IMM и запрещённое моделью OCaml. Полученная схема компиляции даёт корректную схему компиляции OCamlMM в модель Power. Кроме того, при таком подходе доказать корректность компиляции OCamlMM в другую архитектуру можно, доказав корректность компиляции IMМ в эту архитектуру.

Ключевые слова: слабые модели памяти; корректность компиляции; многопоточность; OCaml memory model; IMM.

Для цитирования: Намаконов Е.С., Подкопаев А.В. Компиляция модели памяти OCaml в PowerТруды ИСП РАН, том 31, вып. 5, 2019 г., стр. 63-78. DOI: 10.15514/ISPRAS-2019-31(5)-4

Благодарности: Авторы благодарят коллег по научной группе за участие в обсуждении ранних версий статьи.

7-9, Universitetskaya embankment, St Petersburg, Russia, 199034.

${ }^{2}$ National Research University "Higher School of Economics»,

3 A bld. 1, Kantemirovskaya st., St Petersburg, Russia, 194100. ${ }^{3}$ JetBrains Research,

2, Kantemirovskaya st., St Petersburg, Russia, 197342.

${ }^{4}$ Max Planck Institute for Software Systems,

G26, Paul-Ehrlich st., Kaiserslautern, Germany, 67663.

Abstract. The development of memory models aimed at solving various concurrency problems is an active research topic. One such model is the OCaml memory model (OCamlMM), which allows to mitigate undefined behavior caused by data races. To use this model in practice one has to prove the correctness of its compilation into mainstream CPU architectures. At the moment, it is done for x86 and ARM but not for Power.One way to prove the correctness of compilation of OCamlMM into the Power model is to develop a compilation scheme from OCamlMM into the Intermediate Memory Model (IMM). It would be sufficien since there already exists a compilation scheme from IMM to the Power model. In this paper, the compilation scheme from OCamlMM into IMM is presented and proved to be correct. Memory fences an compare-and-swap instructions are used to permit only behavior allowed by OCamlMM. The resulting compilation scheme gives a correct compilation scheme from OCamlMM to the Power model. Moreover when a compilation scheme from IMM into another CPU architecture will be developed, such an approach would immediately give a correct scheme of compilation of OCamIMM into that architecture.

Keywords: weak memory models; compilation correctness; concurrency; OCaml memory model; IMM

For citation: Namakonov E. S., Podkopaev A. V. Compilation of OCaml memory model into Power. Trudy ISP RAN/Proc. ISP RAS, vol. 31, issue 5, 2019, pp. $63-78$ (in Russian). DOI: 10.15514/ISPRAS-201931(5)-4

Acknowledgements. Authors are grateful to the research group colleagues for participating in early paper versions' discussions.

\section{1. Введение}

Семантика языка программирования, поддерживаюшего параллельные вычисления, определяет множество возможных состояний системы (главным образом - оперативной памяти) после выполнения программы посредством модели памяти. Наиболее известная модель памяти - модель последовательной согласованности (sequential consistency, SC [1]), в которой любой результат исполнения программы может быть получен путём попеременного исполнения инструкций отдельных потоков согласно программному порядку в них. Однако из-за оптимизаций, выполняемых компилятором и процессором, могут наблюдаться поведения, невозможные в такой модели. Например, при выполнении программы ${ }^{1}$ на рис. 1 , написанной на $\mathrm{C}++$ и скомпилированной с помощью компилятора GCC, на архитектуре х86 оба потока могут прочитать значение 0, что объясняется буферизацией записи (store buffering, [2] [3]).

Так как отказ от подобных оптимизаций нежелателен, современные модели памяти допускают некоторые сценарии поведения, невозможные в модели SC. Такие модели памяти называются слабыми. Например, слабыми являются модели памяти языков C++

Здесь и далее используется упрощённый синтаксис программ: $x$ и $y$ обозначают адреса в памяти, $a$ и $b-$ локальные переменные (регистры), $r l x$ - режим доступа. В комментариях указаны наблюдаемые при чтении 

[12] [13].

\begin{tabular}{l||c}
\hline \multicolumn{2}{c}{$x=y=0$} \\
\hline$[x]^{r l x}:=1 ;$ & {$[y]^{r l x}:=1 ;$} \\
$a:=[y]^{r l x} ; / / 0$ & $b:=[x]^{r l x} ; / / 0$
\end{tabular}

\section{Рис. 1. Буферизаиия записи} Fig. 1. Store buffering

Так как отказ от подобных оптимизаций нежелателен, современные модели памяти допускают некоторые сценарии поведения, невозможные в модели SC. Такие модели памяти называются слабыми. Например, слабыми являются модели памяти языков С++ [4], Java [5] и JavaScript [6] [7], а также архитектур х86 [2] [8], Power [9] [10]и ARM [11] [12] [13]. Для усиления гарантий на поведение программы в слабой модели памяти необходимо использовать инструкции с более строгим режимом доступа: например, модель памяти $\mathrm{C}++$ гарантирует, что если в программе на рис. 1 заменить режим доступа всех инструкций с $r l x$ на $s c$, то поведение полученной программы будет согласовано с моделью SC, поскольку такие инструкции будут скомпилированы с использованием т.н. барьеров памяти, запрещающих перестановку инструкций программы.

Одной из проблем таких моделей памяти является то, что они предоставляют слабые гарантии на поведение программ, содержащих гонки по данным. В частности, в модели C++ поведение таких программ не определено (см. [4], раздел 2). А модель памяти Java допускает чтение произвольных значений по отдельному адресу, если раньше по нему произошла гонка (см. [14]).

Для решения этой проблемы была предложена модель памяти OCaml (далее - OCamlMM) [15], обладающая свойством локальной свободы от гонок (Local Data Race Freedom property): результат обращения по данному адресу в памяти не зависит от гонок по другим адресам, а также от предыдущих гонок по этому же адресу. Это свойство гарантирует, что результат исполнения всех участков программы, не содержащих гонок по данным, будет согласовано с моделью SC.

Для того, чтобы использовать ОCamlMM на практике, необходимо доказать её реализуемость на распространённых архитектурах процессоров. Для этого нужно доказать корректность компиляции в каждую из них - показать, что для любой программы при замене инструкций языка на процессорные инструкции согласно схеме компиляции получается программа, все сценарии поведения которой разрешены ОСаm1MM для исходной программы. В [15] приведены схемы компиляции ОСamlMM в модели х86 и ARMv8 и доказана их корректность. При этом отсутствует схема компиляции в модель Power - архитектуру, часто используемую в серверном оборудовании [16]. Задача построения такой схемы осложнена тем, что модель Power, в отличие от моделей х86, ARMv8 и OCam1MM, не обладает т.н. свойством multicopy atomicity, т.е. не гарантирует, что записанные в память значения становятся доступны всем потокам в одном и том же порядке [11]

Для доказательства корректности компиляции OCamlMM в модель Power достаточно построить корректную схему компиляции ОСamlMM в промежуточную модель памяти (Intermediate Memory Model, далее - IMM) [17], для которой уже доказана корректность компиляции в модель Power. Использование IMM как промежуточного этапа компиляции позволяет разбить доказательство корректности компиляции модели языка в модель архитектуры на два, которые впоследствии можно использовать в других доказательствах для этих моделей.
Namakonov E. S., Podkopaev A. V. Compilation of OCaml memory model into Power. Trudy ISP RAN/Proc. ISP RAS, vol. 31, issue 5,

В данной работе предлагается схема компиляции ОCamlMM в IMM и доказывается её корректность. Так как для IMM корректность компиляции в модель Power уже доказана полученная схема даёт корректную схему компиляции ОCam1MM в модель Power.

Статья имеет следующую структуру. Разд. 2 описывает проблему корректности компиляции ОСаmIMM в IMM на примерах. В разд. 3 приводится определение графа исполнения - способа представить исполнение программы в декларативных моделях памяти, к которым относятся ОСаmIMM и IMМ. Затем, в разд. 4 описываются формальные модели OCamIMM и IMM. В разд. 5 и 6 предлагается схема компиляции ОСаmIMM в IMM и доказывается её корректность. В разд. 7 приводится обзор связанных работ. Наконец, в разд. 8 подводятся итоги работы и описываются направления дальнейших исследований.

\section{2. Корректность компиляции ОСатIMM в IMM на примерах}

OCamIMM и IMM определены декларативно. Это означает, что исполнение программы представляется в виде т.н. графа исполнения. Пример программы и одного из графов её исполнения приведён на рис. 2.

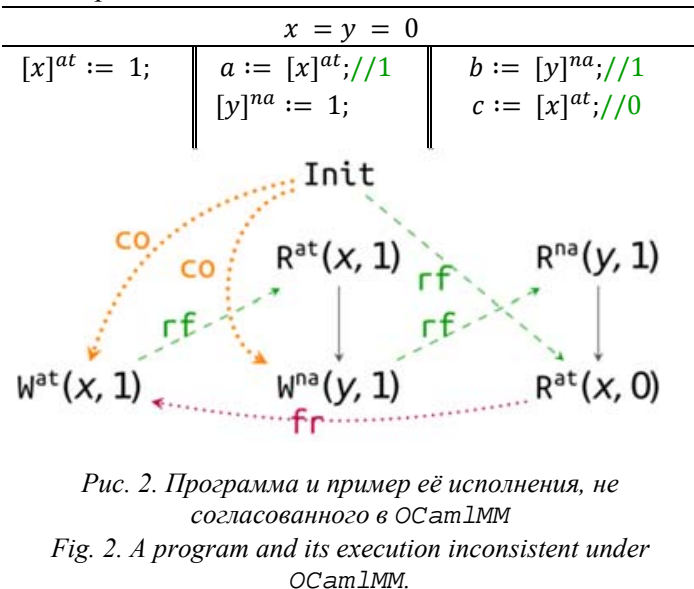

Вершины графа соответствуют событиям - операциям над памятью, которые производятся при выполнении инструкций программы. Так, событие $W^{a t}(x, 1)$ соответствует записи по адресу $x$ значения 1 в режиме $a t$. Кроме того, в графе выделяются инициализирующие события, которые соответствуют инициализирующей записи нулей в память. На рис. 2 они для краткости объединены в множество Init; далее в графах мы будем опускать эти события, если это не будет важно для рассуждений.

Рёбра графа задают бинарные отношения между событиями. В данном графе есть четыре различных отношения: рёбра ро соответствуют программному порядку инструкций, $r f$ чтению записанного ранее значения, со - порядку записей по одному адресу, $f r$ чтению до указанного события записи. Отношения ро и со являются транзитивными, поэтому для их задания достаточно указывать только непосредственные рёбра. Кроме того, для краткости будем опускать подпись "ро" рядом с соответствующими рёбрами.

Согласованными (допустимыми моделью) называются те исполнения, графы которых удовлетворяют некоторому предикату, заданному моделью. В частности, предикат согласованности ОСаmIMM требует, чтобы в графе не было циклов, состоящих только из рёбер $c o$ и $f r$, проходящих между вершинами с меткой $a t$, а также рёбер $p o$ и $r f$. Это условие формализует свойство multicopy atomicity, описанное выше. 
Граф исполнения на рис. 2 не является ОСатlММ-согласованным. Действительно, это исполнение нарушает свойство multicopy atomicity: второй поток читает записанное в $x$ значение 1 до записи 1 в $y$, однако третий поток читает старое значение 0 из $x$ после чтения 1 из $y$. Соответствующий граф исполнения не удовлетворяет предикату согласованности OCamlMM, так как между вершинами есть цикл, подходящий под описание выше. Таким образом, в 0СаmIMM после исполнения программы на рис. 1 переменные $a, b$ и $c$ не могут содержать значения 1,1 и 0 соответственно

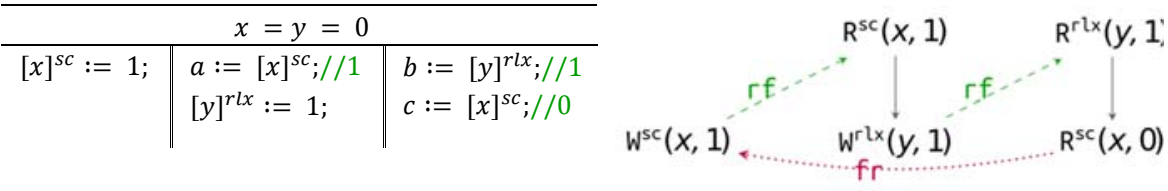

Рис. 3. Результат компиляции программы на рис. 2 с использованием тривиальной схемы компилячии и его IММ-согласованный граф исполнения

Fig. 3. The result of compilation of the program from fig. 2 using the trivial compilation scheme and

$$
\text { its IMM-consistent execution graph }
$$

Скомпилированная программа не должна демонстрировать поведение, запрещённое исходной моделью памяти. Поэтому граф исполнения скомпилированной программы не должен быть согласованным в целевой модели памяти.

На рис. 3 показана программа, полученная в результате компиляции программы на рис. 2 согласно тривиальной схеме компиляции, и граф её исполнения. Такая схема лишь заменяет режимы инструкций на их аналоги в IMM : na заменяется на $r l x$, а $a t-$ на $s c$; дополнительных инструкций не вводится. Соответственно, граф на рис. 3 отличается от графа на рис. 2 только метками вершин, и в нём сохраняется цикл того же вида. IMM не гарантирует свойство multicopy atomicity, и потому предикат её согласованности не требует отсутствия таких циклов, что делает граф на рис. 3 IMМ-согласованным, а соответствующее ему поведение - разрешённым. Поэтому тривиальная схема компиляции не является корректной.

На рис. 4 приведена программа, полученная в результате компиляции программы на рис. 2 согласно схеме компиляции, приведённой в разделе 5 . В результате компиляции в ней появляются инструкции барьеров памяти, запрещающие некоторые оптимизации процессора и компилятора. С ними граф исполнения перестаёт быть согласованным: из рёбер $f r, p o$, окружённого барьерами $r f$, а также $r f$ между событиями с меткой $s c$ образуется цикл, запрещённый в IMM.

\begin{tabular}{l||l||l}
\hline \multicolumn{2}{c|}{$x=y=0$} \\
\hline fence $^{a c q} ;$ & fence ${ }^{a c q} ;$ & $b:=[y]^{r l x} ; / / 1$ \\
exchg $(x, 1) ;$ & $a:=[x]^{\text {sc }} ; / / 1$ & fence $e^{a c q} ;$ \\
& fence ${ }^{\text {acqrel }} ;$ & $c:=[x]^{s c} ; / / 0$ \\
& {$[y]^{r l x}:=1 ;$} &
\end{tabular}

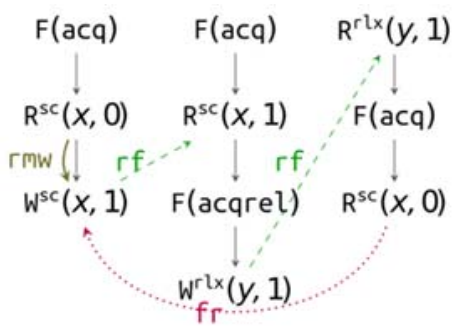

Рис. 4. Результат компиляции программы на рис. 2 с использованием тривиальной схемы компилячии и его IММ-согласованный граф исполнения

Fig. 4. The result of compilation of the program from fig. 2 using the trivial compilation scheme and its IMM-consistent execution graph.
Namakonov E. S., Podkopaev A. V. Compilation of OCaml memory model into Power. Trudy ISP RAN/Proc. ISP RAS, vol. 31, issue 5,

Таким образом, для доказательства корректности компиляции необходимо доказать следующую теорему.

Теорема 2.1. Пусть $G_{I}$ - IMМ-согласованный граф исполнения, соответствующий графу исполнения $G_{0}$ в OCam1MМ. Тогда $G_{0}$ является ОCam1MМ-согласованнылм.

Для доказательства теоремы достаточно доказать ОСаm1MM-согласованность графа $G_{I}$. Из этого следует 0Cam1MM-согласованность графа $G_{O}$, так как он фактически является подграфом $G_{I}$, а условия OCamlMM-согласованности графа таковы, что они выполняются для любого его подграфа. Условие ОСаmІММ-согласованности состоит в иррефлексивности одного отношения и ацикличности другого. Для каждого из этих отношений доказывается включение в такое отношение, для которого в IMMсогласованном графе соответствующее условие выполняется.

\section{3. Понятие графов исполнения}

В описаниях декларативных моделей памяти часто встречаются следуюшие обозначения отношений между вершинами. Для бинарного отношения $R$ обозначения $R$ ? $R^{+}, R^{*}$ соответствуют его рефлексивному, транзитивному и транзитивно-рефлексивному замыканиям соответственно. Обратное отношение записывается как $R^{-1}$, а области определения и значений $-\operatorname{dom}(R)$ и $\operatorname{codom}(R)$. Тождественное отношение на множестве $A$ обозначается как $[A]$, что часто используется в обозначениях вида $[A] ; R ;[B] \triangleq R \cap$ $(A \times B)$. Левая композиция отношений записывается как $R 1 ; R 2 \triangleq\{x, y \mid \exists z .(x, z) \in$ $R 1 \wedge(z, y) \in R 2\}$. Непосредственные рёбра $R$ обозначаются как $\left.R\right|_{\text {imm }} \triangleq R \backslash(R ; R)$.

В данном разделе описываются графы исполнения в наиболее общем виде, без привязки к конкретным моделям памяти или языкам.

Считаем, что анализируемая программа $P$ состоит из последовательных подпрограмм отдельных потоков $P_{i}: P=\|_{i \in T i d} P_{i}$, где $\|-$ оператор параллельной композиции программ, Tid - конечное множество идентификаторов потоков.

Определение 1. Граф исполнения $G$ задаётся множеством вершин $G . E$, отображением $G . L a b$, задающим параметры операций над памятью, и бинарными отношениями на вершинах.

Множество G.E делится на инициализирующие события вида Init loc и неинициализирующие события вида ThreadEvent tid $n$, где:

- $\quad l o c \in L o c$ - адрес инициализации, где $L o c$ - конечное множество адресов;

- $\quad i \in$ Tid-номер потока;

- $n \in Q$ - порядковый номер внутри потока (нумерация плотным порядком упрощает формальное определение соответствия графов, см. раздел 5).

Обозначения e.tid и e.n для $e=$ ThreadEvent tid $n$ соответствуют tid и $n$ соответственно.

Функция G. Lab сопоставляет событиям метки вида $t y p e^{\text {mode }}$ (loc, val), где:

- type $\in\{R, W, F\}$ - тип операции (чтение, запись, барьер);

- mode - один из режимов доступа, частично упорядоченных отношением “строже чем" (コ); конкретное множество режимов и их порядок определяется моделью памяти;

- $\quad l o c \in L o c$ - адрес памяти (для барьера не определено);

- $v a l \in V a l-$ прочитанное/записанное значение (в случае барьера не определено), где $\mathrm{Val}$ - множество значений, которые могут храниться в памяти.

При этом инициализирующие события обрабатываются особым образом: G.Lab(Init loc $)=W^{\text {mode }_{\text {init }}}\left(l o c, v_{\text {al }}\right.$ init $)$, где mode $_{\text {init }}{ }^{-}$режим доступа для 
Введём обозначения для множеств событий с определёнными метками: например, события с меткой чтения в режиме $a c q$ или более строгим будем обозначать как $G . R^{a c q}$ (или просто $R^{a c q}$, если граф очевиден из контекста).

Рёбра графа представляют собой отношения между событиями:

- программный порядок (program order): $G . p o(x, y) \Leftrightarrow(x \in$ Init $\wedge y \notin$ Init) $\vee$ (x.tid $=$ y.tid $\wedge x . n<y . n)$;

- порядок согласованности (coherence order): $G . c 0=\bigcup_{l \in L o c} C_{l}$, где $c_{l}-$ тотальный порядок на событиях записи по адресу $l$;

- наблюдение записанного значения (reads from, «читает-из»): G.rf $\subseteq \mathrm{U}_{l \in L o c} G . W_{l} \times$ $G . R_{l}$, где $G \cdot r f(w, r) \Rightarrow G \cdot L a b(w) \cdot v a l=G \cdot L a b(r) \cdot v a l, \operatorname{codom}(G . r f)=G \cdot R \quad$ и $G . r f$ является функциональным отношением;

- $\quad$ чтение до указанной записи: $G . f r=G . r f^{-1} ; G . c o$ (from-read, «читает-перед»).

В различных моделях памяти в граф исполнения могут добавляться другие отношения. Например, в IMM также есть отношение $r m w \subseteq \cup_{l \in L o c}\left[G . R_{l}\right] ;\left.p o\right|_{i m m} ;\left[G . W_{l}\right]$ соответствующее паре событий чтения и записи в операции read-modify-write.

Введём понятие сужения графа на поток $i: G_{i} . E=\{e \in G . E \mid e . t i d=i\}, G_{i} . L a b=G . L a b$

Определение 2. Графом исполнения программы $P$ называется такой граф исполнения $G$, что его сужение на любой поток $i$ является однопоточным графом исполнения программы $P_{i}$. Соответствие подпрограммы потока и однопоточного графа исполнения определяется средствами операционной семантики, специфичной для языка ( [15], [17], [18]). Мы не приводим подробностей здесь, скажем лишь, что такая семантика задаёт соответствие между выполнением инструкций языка и изменением графа исполнения. Так, для IMM выполнение инструкции $[x]^{r e l}:=1$ соответствует добавлению в текущий граф вершины с очередным порядковым номером и меткой вида $W^{r e l}(x, 1)$ и рёбер, отражающих синтаксические зависимости данной записи.

Определение 3. Результатом работы графа G называется функция $f:$ Loc $\rightarrow \mathrm{Val}$, отображающая адрес в последнее (согласно порядку со) записанное по нему значение. Декларативная модель памяти задаётся предикатом согласованности, которому должны удовлетворять графы исполнения программ.

Определение 4. Результатом работы программы $P$ в модели памяти $M$ является результат работы некоторого графа её исполнения, удовлетворяющего предикату согласованности $M$.

\section{4. Описание используемых моделей памяти}

В данном разделе описываются рассматриваемые модели памяти и их предикаты согласованности.

\subsection{OCaml Memory Model}

OCamIMM задана в [15] эквивалентными операционным и декларативным описаниями. Для доказательства корректности компиляции будет использоваться декларативное описание.

oCamIMM поддерживает два режима доступа: неатомарный $n a$ и атомарный at (схожи c $p l n$ и $s c$ в C++). При этом память также разделена на неатомарные и атомарные адреса, и к конкретному адресу можно обратиться только операцией соответствующего режима. В графе исполнения ОСаmIMM есть только операции чтения и записи, барьеры отсутствуют.
Namakonov E. S., Podkopaev A. V. Compilation of OCaml memory model into Power. Trudy ISP RAN/Proc. ISP RAS, vol. 31, issue 5,

Перед рассмотрением предиката согласованности введём ещё несколько обозначений Для отношения $R$ будем обозначать $R i$ рёбра $R$, проходящие между вершинами одного потока, а $R e$-между вершинами разных потоков.

Определение 5. Исполнение называется 0CamlMM-согласованным, если в соответствующем графе исполнения выполняются следующие аксиомы:

1) отношение $h b o$; $(c o \cup f r)$ иррефлексивно, где $h b o \triangleq p o \cup\left[E^{a t}\right] ;(c o \cup r f) ;\left[E^{a t}\right]$

2) отношение po $\cup$ rfe $\cup\left[E^{a t}\right]$; (coe $\cup$ fre); $\left[E^{a t}\right]$ ациклично.

\subsection{Intermediate Memory Model}

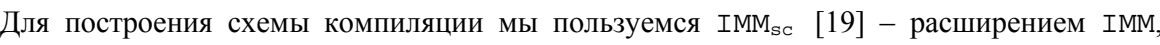
которое дополняет оригинальную модель [17] SC-операциями.

IMM определена декларативно. Полный предикат согласованности IMM достаточно сложен, поэтому мы рассмотрим лишь часть модели, которая будет необходима для построения схемы компиляции.

Синтаксис программ в IMM напоминает таковой в $\mathrm{C}++-$ помимо инструкций атомарного чтения и записи есть инструкции барьеров памяти, а также операций read-modify-write. Пары событий чтения и записи, порождаемых инструкциями read-modify-write, связань отношением $r m w \subseteq\left([G . R] ;\left.p o\right|_{i m m} ;[G . W]\right)_{l o c}$. Доступно несколько режимов доступа, упорядоченных следующим образом: $ᄃ \triangleq\{(r l x, a c q),(r l x, r e l)$, (acq, acqrel), (rel, acqrel), (acqrel, sc)\}.

Введём ещё несколько обозначений. $R_{l o c}$ будем обозначать рёбра $R$, проходящие между вершинами с метками одного и того же адреса, $R_{\neq l o c}-$ между вершинами с метками разных адресов.

Определение 6. Исполнение называется ІММ-согласованным, если в соответствующем графе исполнения выполняются следующие аксиомы:

1) отношение $h b ;(r f \cup c o \cup f r)^{+}$иррефлексивно, где $h b \triangleq(p o \cup s w)^{+}$

$s w \triangleq$ release; $\left(r f i \cup p o^{?}{ }_{l o c} ; r f e\right) ;\left(\left[R^{a c q}\right] \cup p o ;\left[F^{a c q}\right]\right)$

release $\triangleq\left(\left[W^{r e l}\right] \cup\left[F^{r e l}\right] ; p o\right) ; r s$

$r s \triangleq[W] ; \mathrm{po}_{l o c} ;[W] \cup[W] ;\left(\mathrm{po}^{?}{ }_{l o c} ; \mathrm{rfe} ; \mathrm{rmw}\right)^{*}$

2) операции read-modify-write являются атомарными: $r m w \cap($ fre; coe $)=\varnothing$

3) отношение $a r$ ациклично, где

ar $\supset$ rfe $\cup$ bob

$b o b \supset\left[R^{a c q}\right] ; p o \cup p o ;[F] \cup[F] ; p o$

4) отношение $p s c$ base ациклично, где $p_{\text {base }} \triangleq\left(\left[E^{s c}\right] \cup\left[F^{s c}\right] ; h b^{?}\right) ; s c b ;\left(\left[E^{s c}\right] \cup h b^{?} ;\left[F^{s c}\right]\right)$ $s c b \triangleq p o \cup p o_{\neq l o c} ; h b ; p o_{\neq l o c} \cup h b_{l o c} \cup c o \cup f r$

\section{5. Схема компиляцич}

Предлагаемая схема компиляции ОCamlMM в IMM описана в табл. 1. За основу взята схема компиляции OCamIMM в модель ARM из [15]. 


\begin{tabular}{|c|c|}
\hline \multicolumn{2}{|c|}{$\begin{array}{l}\text { Табл. 1. Схема компиляиии модели OCaml в } \\
\text { nромежуточную модель. } \\
\text { Table 1. Scheme of compilation of OCaml model into IMM }\end{array}$} \\
\hline OCamlMM & IMM \\
\hline $\begin{array}{l}r:=[x]^{n a} \\
{[x]^{n a}:=v} \\
r:=[x]^{a t} \\
{[x]^{a t}:=v}\end{array}$ & $\begin{array}{c}r:=[x]^{r l x} \\
\text { fence }^{\text {acqrel }} ;[x]^{r l x}:=v \\
\text { fence }^{a c q} ; r:=[x]^{s c} \\
\text { fence }^{\text {acq }} ; \operatorname{exchg}(x, v)\end{array}$ \\
\hline
\end{tabular}

Напомним, что схема компиляции корректна, если все возможные сценарии поведения скомпилированной программы являются допустимыми сценариями поведения исходной программы согласно исходной модели памяти. В случае декларативных моделей памяти это можно переформулировать так: если граф исполнения скомпилированной программы согласован с целевой моделью памяти, то соответствующий ему граф исполнения исходной программы согласован с исходной моделью памяти.

Чтобы формализовать понятие соответствия графов, опишем, чем отличаются графы исполнения скомпилированной программы и исходной. Во-первых, все вершины исходного графа сохраняются, а новые вершины добавляются согласно схеме компиляции. Во-вторых, порядок согласованности сохраняется, так как события записи в новом графе те же, что и в исходном. В-третьих, при компиляции используются инструкции CAS, поэтому в графе появляется отношение $r m w$. Наконец, так как инструкции CAS в графе выражаются с помощью пар чтения и записи, в отношении "читает-перед" появляются новые рёбра, которые указывают на значения, наблюдаемые при исполнении CAS. Формально эти условия описываются следующим образом.

Для краткости события вида ThreadEvent in будем обозначать как $\langle i, n\rangle$.

Определение 7. Граф исполнения по ОСаmIMM $G_{O}$, события в потоках которого пронумерованы натуральными числами, соответствует графу исполнения по IMM $G_{I}$, если выполняются следующие условия:

1) $G_{I} \cdot E=G_{O} \cdot E \cup\left\{\langle i, n-0.5\rangle \mid\langle i, n\rangle \in G_{O} \cdot\left(E \backslash R^{n a}\right)\right\} \cup\{\langle i, n-0.25\rangle \mid\langle i, n\rangle \in$ $\left.G_{O} . W^{a t}\right\}$

2) $\left.G_{I} . L a b=\{e \rightarrow(t, l, \operatorname{rename}(m), v)) \mid G_{O} . \operatorname{Lab}(e)=(t, l, m, v)\right\}$

$\cup\left\{\langle i, n-0.5\rangle \rightarrow(F,-, a c q,-) \mid\langle i, n\rangle \in G_{0} \cdot E^{a t}\right\}$

$\cup\left\{\langle i, n-0.5\rangle \rightarrow(F,-\right.$, acqrel,-$\left.) \mid\langle i, n\rangle \in G_{O} . W^{n a}\right\}$

$\cup\left\{\langle i, n-0.25\rangle \rightarrow(R, l, s c, v) \mid\langle i, n\rangle \in G_{O} . W^{a t} \wedge G_{O} . L a b(\langle i, n\rangle)=\right.$ $(W, l, a t,-) \wedge v \in V a l\}$,

где $\operatorname{rename}(n a)=r l x$,rename $(a t)=s c$

3) $G_{I} . r m w=\left\{(r, w) \mid w \in G_{I} . W^{s c} \wedge r \in G_{I} . R^{s c} \wedge r . t i d=w . t i d \wedge r . n=w . n-\right.$ $0.25\}$

4) $G_{I} \cdot C O=G_{O} \cdot C O$

5) $G_{I} \cdot r f \supset G_{O} \cdot r f$.

Видно, что нумерация событий в потоках рациональными числами позволяет добавлять в граф новые события, по программному порядку находящиеся между существующими.

Будем рассматривать граф исполнения скомпилированной IMМ-программы как граф исполнения исходной ОСamIMM-программы. В самом деле, при компиляции лишь добавляются новые вершины и изменяются метки у существующих. Выполнив обратное преобразование, можно получить исходный граф исполнения в OCamlMM. Поэтому для
Namakonov E. S., Podkopaev A. V. Compilation of OCaml memory model into Power. Trudy ISP RAN/Proc. ISP RAS, vol. 31, issue 5,

графа исполнения в IMM можно анализировать также и его согласованность по ОСаmIMM, заменяя в предикате согласованности ОСamlMM режимы с $n a$ на $r l x$ и с at на $s c$.

Теперь можно объяснить выбор данной схемы компиляции: использование инструкций compare-and-swap и барьеров накладывает на исполнение программы в IMM условия, достаточно строгие для выполнения в согласованном по IMM графе первого и второго условий согласованности по ОСаmIMM соответственно.

Рассмотрим несколько примеров, демонстрирующих необходимость расположения барьеров.

Рис. 5 демонстрирует, что при компиляции инструкции неатомарной записи необходимо использовать именно барьер в режиме acqrel, а не rel. Такая оптимизация может показаться разумной, т.к. $S W$ (и, следовательно, $h b$ ) в IMM может начинаться с $F^{r e l}$. Описанное поведение должно быть запрещено, так как в графе есть запрещённый в OCamIMM цикл $p o ; r f ; p o ; r f ; p o ;\left[E^{s c}\right] ; f r ;\left[E^{s c}\right]$. Однако IMM разрешает такое поведение, так как после первого ребра $r f$ нет ни $R^{a c q}$, ни $F^{a c q}$. Если бы вместо $F^{r e l}$ во втором потоке располагался $F^{a c q r e l}$, как это предполагает схема компиляции, то через первое ребро $r f$ прошёл бы $h b$, и результирующий граф был бы запрещён IMM.

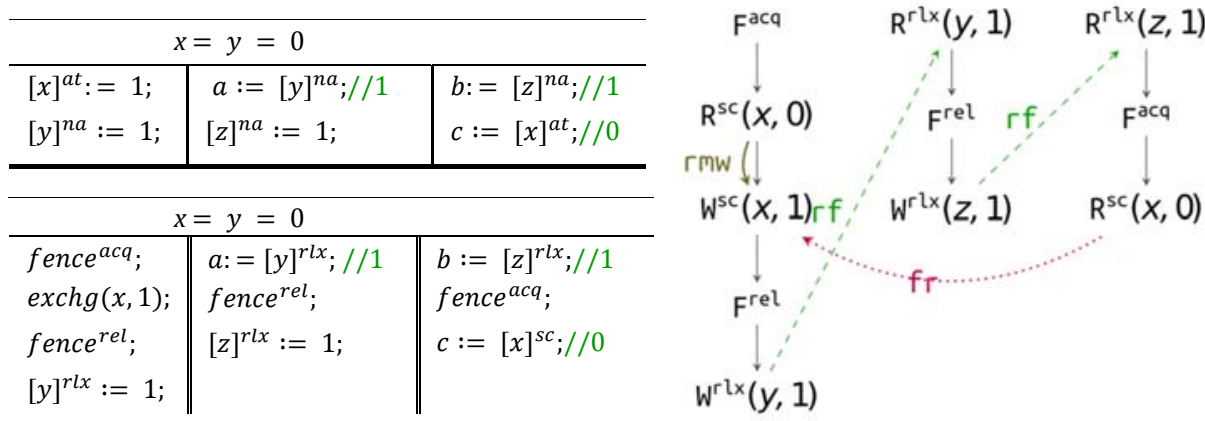

Рис. 5. Пример программы, результата её компиляиии схемой с rel-барьером перед инструкииями $r l x$-записи и ІММ-согласованного графа её исполнения

Fig. 5. An example of a program, the result of its compilation with a scheme using rel fence before rlx write instructions and its IMM-consistent execution graph.

Рис.6 демонстрирует, что барьер перед CAS удалить нельзя. В нём есть цикл $p o ; r f ; p o ; r f ; p o ;\left[E^{s c}\right] ; f r$, запрешённый в OCamIMM, но разрешённый в IMM: SW должен оканчиваться либо $R^{a c q}$, либо $R$ с последующим барьером. Ни того, ни другого во втором потоке нет, поэтому между первым и вторым потоком нельзя проложить $h b$. Если перед $R^{s c}$ во втором потоке расположить $a c q$-барьер, то результирующий граф будет также запрещён и IMM.

\section{6. Доказательство корректности компиляции}

Напомним формулировку теоремы о корректности компиляции.

Теорема 2.1. Пусть $G_{I}$ - IMМ-согласованный граф исполнения, соответствующий графу исполнения $G_{O}$ в OCamlMM. Тогда $G_{O}$ является OCamlMM-согласованным.

Как было показано выше, OCam1MM-согласованность $G_{O}$ следует из 0Сam1MMсогласованности $G_{I}$. Поэтому далее будем доказывать два условия 0Саm1MMсогласованности для $G_{I}$. 


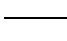

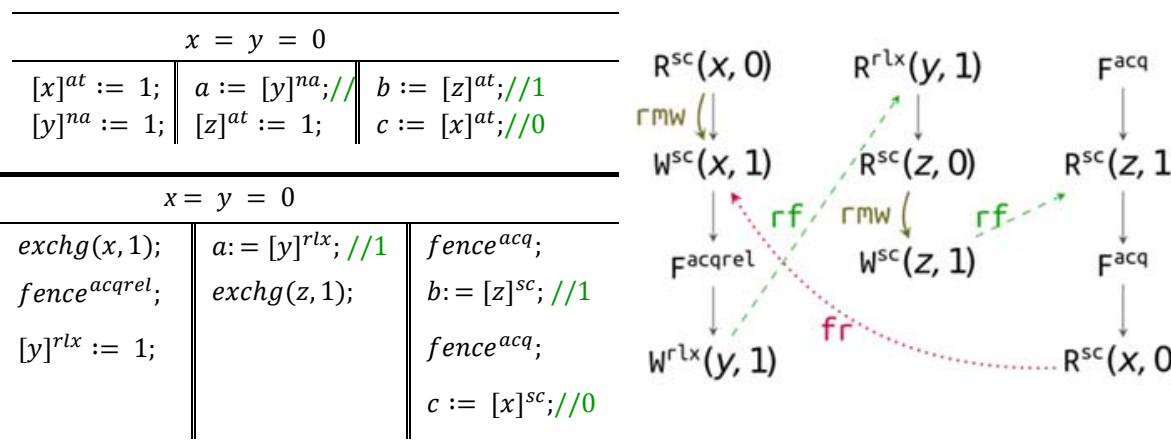

Рис. 6. Пример программы, результата её компиляиии схемой, не использующей барьер перед инструкииями сотраге - and - swap, и IMM-согласованного графа его исполнения

Fig. 6. An example of a program, the result of its compilation with a scheme not using a fence before compare - and - swap instructions and its IMM-consistent execution graph.

\section{1. Первое условие ОСатІММ-согласованности}

Теорема 6.1. Отношение $h b o$; (co $\cup f r$ ) иррефлексивно.

Докажем, что с выбранной схемой компиляции $h b o \subseteq h b$. С учётом этого доказательство теоремы тривиально: согласно ІММ-согласованности, отношение $h b ;(r f \cup c o \cup f r)$ иррефлексивно.

Для этого сначала покажем, что последовательность $c o$ по $s c$-событиям порождает $h b$. Лемма 6.2. Порядок Sc-записей согласуется с отношением happens-before:

$\left[E^{s c}\right] ; c 0 ;\left[E^{s c}\right] \subseteq h b$.

Доказательство. Заметим, что $\left[E^{s c}\right] ; c 0 ;\left[E^{s c}\right]$ транзитивно. Тогда можно перейти к рассмотрению непосредственных $c 0$-соседей.

Предположим, что $\left[E^{s c}\right] ;\left.c o\right|_{i m m ;}\left[E^{s c}\right] \subseteq\left[E^{s c}\right] ; r f ;\left[E^{s c}\right] ; p o$. Тогда доказательство тривиально: $r f$ по $s c$ событиям порождает $h b$, как и следующий за ним ро. Значит, остаётся доказать утверждение о включении в $\left[E^{s c}\right] ; r f ;\left[E^{s c}\right] ;$ ро.

Рассмотрим два события $w 1, w 2 \in W^{s c}$ - непосредственных со-соседей. По схеме компиляции перед $w 2$ следуют $f \in F^{a c q}$ и $r \in R^{s c}$, причём $r m w(r, w 2)$.

Покажем, что $r f(w 1, r)$. В самом деле, рассмотрим событие записи $w^{\prime}$, из которого читает $r$. Так как обращения по одному и тому же адресу имеют один и тот же режим, то $w^{\prime} \in$ $W^{s c}$.

Пусть $w 1 \neq w^{\prime}$. Тогда либо $c o\left(w^{\prime}, w 1\right)$, либо, наоборот, $c o\left(w 1, w^{\prime}\right)$. В первом случае нарушается атомарность $r m w$ между $w 2$ и $r$. Во втором случае

получается, что между со-соседями $w 1$ и $w 2$ расположен $w^{\prime}$, что невозможно. $\square$

Лемма 6.3. Отношение happens-before в ОСаmIMM является подмножеством happensbefore в IMM: $h b o \subseteq h b$.

Доказательство. $h b o \triangleq p o \cup\left[E^{s c}\right] ;(c o \cup r f) ;\left[E^{s c}\right]$. По предыдущей лемме co, ограниченный на $s c$, входит в $h b$.

$r f$ по $s c$ событиями порождает $s w$, и, следовательно, $h b . \square$

Таким образом, $h b o \subseteq h b$, из чего, согласно IMМ-согласованности, следует, что $h b o ;(c o \cup f r)$ иррефлексивно. $\square$
Namakonov E. S., Podkopaev A. V. Compilation of OCaml memory model into Power. Trudy ISP RAN/Proc. ISP RAS, vol. 31, issue 5,

\section{2. Второе условие ОСаmIMM-согласованности}

Сначала докажем утверждения, которые позволят нам находить барьеры в программном порядке между событиями.

Лемма 6.4. В программном порядке между произвольным событием и записью располагается барьер: $\left.\left[F^{a c q}\right] ; p o ;\left[E^{s c}\right]\right) ;[W] \cup r m w$.

$[E \backslash F] ; p o ;[W] \subseteq p o ;\left(\left[F^{a c q r e l}\right] ; p o ;\left[E^{r l x}\right] \cup\right.$

Доказательство. Согласно схеме компиляции, инструкция барьера располагается либо непосредственно перед инструкцией неатомарной записи, либо перед инструкцией compare-and-swap. Таким образом, барьера между событием и ро-следующей записью может не быть, только если это событие чтения в $r m w . \quad \square$

Лемма 6.5. В программном порядке между $r l x$ и $s c$ событиями располагается барьер: $\left[E^{r l x}\right] ; p o ;\left[E^{s c}\right] \subseteq p o ;\left[F^{a c q}\right] ; p o$

Доказательство. Согласно схеме компиляции, все инструкции в режиме $s c$ предваряются $a c q$ барьерами. $\square$

Кроме того, понадобятся следующие факты из алгебры:

Лемма 6.6. Цикл из рёбер двух типов можно представить в виде чередующихся участков рёбер каждого типа: $(x \cup y)^{+}=y^{+} \cup y^{*} ;\left(x ; y^{*}\right)^{+}$, где $x, y$ - произвольные отношения. Лемма 6.7. Отношение $x \cup y$ ациклично, если ацикличны отношения $x, y$ и $x^{+} ; y^{+}$. Наконец, мы можем перейти ко второму условию согласованности по ОСаmIMM.

Теорема 6.8. Отношение po $\cup \mathrm{rfe} \cup\left[E^{s c}\right]$; (coe $\cup$ fre $) ;\left[E^{s c}\right]$ ациклично.

Сгруппируем первые два отношения в объединении. Тогда по лемме 6.7 нужно показать ацикличность следующих отношений:

- po U rfe;

- $\left[E^{s c}\right] ;($ coe $\cup$ fre $) ;\left[E^{s c}\right]$;

- $\quad(\text { ро } \cup r f e)^{+} ;\left(\left[E^{s c}\right] ;(\text { co } \cup \cup f r e) ;\left[E^{s c}\right]\right)^{+}$, что эквивалентно ацикличности $\left[E^{s c}\right] ;(\text { po } \cup \text { rfe e })^{+} ;\left[E^{s c}\right] ;\left(\left[E^{s c}\right] ;(\text { coe } \cup \text { fre }) ;\left[E^{s c}\right]\right)^{+}$

Второе и третье утверждение докажем, показав, что соответствующие отношения лежат в $\left(\left[E^{s c}\right] ; s c b ;\left[E^{s c}\right]\right)^{+} \subseteq p s c$ base $^{+}$, где, напомним, $s c b \triangleq p o \cup p o_{\neq l o c} ; h b ; p o_{\neq l o c} \cup$ hbloc $\cup$ $c o \cup f r \quad$ и $\quad p s c$ base $\triangleq\left(\left[E^{s c}\right] \cup\left[F^{s c}\right] ; h b ?\right) ; s c b ;\left(\left[E^{s c}\right] \cup h b^{?} ;\left[F^{5 c}\right]\right)$. В свою очередь, ацикличность $p S c$ base следует из IММ-согласованности графа.

Теперь видно, что второе утверждение верно по определению $s c b$. По этой же причине для доказательства третьего утверждения будет достаточно показать, что $\left[E^{s c}\right]$; $($ po $\cup$ $r f e)^{+} ;\left[E^{s c}\right] \subseteq\left(\left[E^{s c}\right] ; s c b ;\left[E^{s c}\right]\right)^{*}$

Теорема 6.9. Отношение ро $\mathrm{U}$ rfe ациклично.

Доказательство. Вновь воспользуемся леммой 6.7 и разложим условие ацикличности объединения на ацикличность отношений ро (следует из IMМ-согласованности) и $r f e$ (двух и более таких рёбер подряд идти не может, т.к. их концы имеют разные типы), а также $\mathrm{o}^{+} ; r f e^{+}$, что эквивалентно ацикличности $p o ; r f e$.

Пусть такой цикл существует. Покажем, что это противоречит условию ацикличности $a r$ (что следует из ІММ-согласованности). Напомним, что $a r \supset r f e \cup b o b$ и $b o b$ د $\left[R^{a c q}\right] ; p o \cup$ po; $[F] \cup[F] ; p o$.

По лемме 6.4 перед событием записи, которой начинается ребро $r f e$, есть барьер $F^{\exists a c q}$, либо весь ро является $r m w$. В первом случае внутри ро есть барьер, а такое отношение лежит в $b o b \subseteq a r$. Во втором случае $r m w$ начинается с $R^{s c}$, и такое ребро ро $\supseteq r m w$ также содержится в $b o b$. Наконец, $r f e \subseteq a r . \square$ 


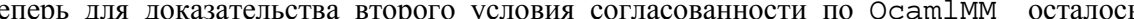
доказать утверждение $\left[E^{s c}\right] ;(\text { o } \cup \mathrm{rfe})^{+} ;\left[E^{s c}\right] \subseteq\left(\left[E^{s c}\right] ; s c b ;\left[E^{s c}\right]\right)^{*}$.

Теорема 6.10. Последовательность из рёбер ро и $r f e$ между вершинами $s c$ состоит из pёбер $s c b$ между вершинами $s c:\left[E^{s c}\right] ;(p o \cup r f e)^{+} ;\left[E^{s c}\right] \subseteq\left(\left[E^{s c}\right] ; s c b ;\left[E^{s c}\right]\right)$

Доказательство. Сначала введём утверждение, которое позволит отбрасывать $r f e$-рёбра. Лемма 6.11. Рёбра $r f e$, у которых один из концов - sc, входят в $s c b:\left[E^{s c}\right] ; r f e U$ $[W \backslash$ Init $] ; r f e ;\left[E^{s c}\right] \subseteq\left[E^{s c}\right] ; s c b ;\left[E^{s c}\right]$.

Доказательство. Если один из концов ребра $r f$ является $s c$, таким же является и второй (исключение-чтение из инициализирующих записей, которые в IMM являются $r l x$ ). Тогда $\left[E^{s c}\right] ; r f ;\left[E^{s c}\right] \subseteq\left[E^{s c}\right] ; h b_{l o c} ;\left[E^{s c}\right] \subseteq\left[E^{s c}\right] ; s c b ;\left[E^{s c}\right]$.

По лемме 6.6 имеем

$\left[E^{s c}\right] ;(p o \cup r f e)^{+} ;\left[E^{s c}\right]=\left[E^{s c}\right] ;\left(r f e^{+} \cup r f e^{*} ;\left(p o ; r f e^{*}\right)^{+}\right) ;\left[E^{s c}\right]=\left[E^{s c}\right] ;(r f e \cup$ $\left.r f e^{?} ;\left(p o ; r f e^{?}\right)^{+}\right) ;\left[E^{s c}\right]$.

Начальные участки $r f e$, если они есть, можно отбросить по лемме 6.11. Рассмотрим оставшееся транзитивное замыкание:

$\left(p o ; r f e^{?}\right)^{+}=p o ;(p o ; r f e)^{*} ; p o^{?}=p o ; p o^{?} \cup p o ;(p o ; r f e)^{+} ; p o^{?}=p o \cup$

$(p o ; r f e)^{+} ; p o^{?}$.

В первом случае отношение сводится к ро $\subseteq s c b$. Во втором, если последним ребром является $r f e$, то его можно отбросить по лемме 6.11. Остаётся случай $(p o ; r f e)^{+} ; p o$. В каждой паре $p o ; r f e$ можно применить лемму 6.4. В результате нужно доказать такое утверждение:

$\left[(W \cup R)^{s c}\right] ;\left(p o ;\left(\left[F^{a c q r e l}\right] ; p o ;\left[E^{r l x}\right] ; r f e \cup\left[F^{a c q}\right] ; p o ;\left[E^{s c}\right] ; r f e\right) \cup\right.$

$r m w ; r f e)^{+} ; p o ;\left[(W \cup R)^{s c}\right] \subseteq\left(\left[E^{s c}\right] ; s c b ;\left[E^{s c}\right]\right)^{*}$.

Воспользуемся леммой 6.6: либо транзитивное замыкание состоит только из пар $r m w ; r f e$, либо такие пары рёбер могут следовать после $p o ;\left(\left[F^{a c q r e l}\right] ; p o ;\left[E^{r l x}\right] ; r f e U\right.$ $\left.\left[F^{a c q}\right] ; p o ;\left[E^{s c}\right] ; r f e\right)$. В первом случае замыкание имеет вид $h b_{l o c} \subseteq s c b$, а так как оно заканчивается $s c$-событием, оставшееся ребро ро также пройдёт по $s c$ и образует $s c b$. Во втором случае рассмотрим, что именно находится под транзитивным замыканием:

(po; $\left.\left(\left[F^{a c q r e l}\right] ; p o ;\left[E^{r l x}\right] ; r f e \cup\left[F^{a c q}\right] ; p o ;\left[E^{s c}\right] ; r f e\right) ;(r m w ; r f e)^{*}\right)^{+}=$

$\left(\left(p o ;\left[F^{a c q}\right] ;\left(\left[F^{a c q r e l}\right] ; p o ;\left[E^{r l x}\right] \cup\left[F^{a c q}\right] ; p o ;\left[E^{s c}\right]\right) ;(r f e ; r m w)^{*} ; r f e\right)^{+}\right)=$

$\left(\left(p o ;\left[F^{a c q}\right] ; C ; r f e\right)\right)^{+}$

где $C=C 1 \cup C 2=\left[F^{a c q r e l}\right] ; p o ;\left[E^{r l x}\right] ;(r f e ; r m w)^{*} \cup\left[F^{a c q}\right] ; p o ;\left[E^{s c}\right] ;(r f e ; r m w)^{*}$.

Заметим, что $\left(p o ;\left[F^{a c q}\right] ; C ; r f e\right)^{+}=p o ;\left[F^{a c q}\right] ;\left(C ; r f e ; p o ;\left[F^{a c q}\right]\right)^{*} ; C ; r f e$. В результате необходимо доказать следующее:

$\left[(W \cup R)^{s c}\right] ; p o ;\left[F^{a c q}\right] ;\left(C ; r f e ; p o ;\left[F^{a c q}\right]\right)^{*} ; C ; r f e ; p o ;\left[(W \cup R)^{s c}\right] \subseteq$

$\left(\left[E^{s c}\right] ; s c b ;\left[E^{s c}\right]\right)^{*}$.

Заметим, что

$\left(C ; r f e ; p o ;\left[F^{a c q}\right]\right)^{*}=\left(\left(\left[F^{a c q r e l}\right] ; p o ;\left[E^{r l x}\right] \cup\right.\right.$

$\left.\left.\left[F^{a c q}\right] ; p o ;\left[E^{s c}\right]\right) ;(r f e ; r m w)^{*} ; r f e ; p o ;\left[F^{a c q}\right]\right)^{*} \subseteq h b^{?}$, так как

$h b \triangleq(p o \cup s w)^{+}$

sw $\supset$ release; $r f e ; p o ;\left[F^{a c q}\right]$,

release $\triangleq\left([\mathrm{Wrel}] \cup\left[\mathrm{F}^{\text {rel }}\right] ;\right.$ po $) ;$ rs ,

rs $\supset(\text { rfe; } r m w)^{*}$.

Вспомним, что $p o_{\neq l o c} ; h b ; p o_{\neq l o c} \subseteq s c b$. Воспользуемся тем, что $p o$ между между событием чтения/записи и барьером образует именно $p o_{\neq l o c}$. Тогда видно, что $\left[(W \cup R)^{s c}\right] ; p o ;\left[F^{a c q}\right] \subseteq\left[E^{s c}\right] ; p o_{\neq l o c}$
Namakonov E. S., Podkopaev A. V. Compilation of OCaml memory model into Power. Trudy ISP RAN/Proc. ISP RAS, vol. 31, issue 5,

Остаётся показать, что $\left[E^{s c}\right] ; p o_{\neq l o c} ; h b ; C ; r f e ; p o ;\left[(W \cup R)^{s c}\right] \subseteq\left(\left[E^{s c}\right] ; s c b ;\left[E^{s c}\right]\right)^{*}$. Для этого перепишем $C=C 1 \cup C 2$ и докажем утверждение для $C 1$ и $C 2$ по отдельности.

- Покажем, что $\left[E^{s c}\right] ; p o_{\neq l o c} ; h b^{?} ; C 1 ; r f e ; p o ;\left[(W \cup R)^{s c}\right] \subseteq\left(\left[E^{s c}\right] ; s c b ;\left[E^{s c}\right]\right)^{*}$.

Заметим, что по лемме 6.5 в последнем ребре ро найдётся $a c q$-барьер, с помощью которого можно будет построить ребро $h b$ :

$C 1 ; r f e ; p o ;\left[(W \cup R)^{s c}\right]$

$=\left[F^{\text {acqrel }}\right] ; p o ;\left[E^{r l x}\right] ;(r f e ; r m w)^{*} ; r f e ; p o ;\left[(W \cup R)^{s c}\right]$

$=\left[F^{a c q r e l}\right] ; p o ;\left[E^{r l x}\right] ;(r f e ; r m w)^{*} ; r f e ;\left[E^{r l x}\right] ; p o ;\left[F^{a c q}\right] ; p o ;\left[(W \cup R)^{s c}\right] \subseteq$ $h b ; p o_{\neq l o c} ;\left[E^{s c}\right]$

Тогда

$\left[E^{s c}\right] ; p o_{\neq l o c} ; h b^{?} ; C 1 ; r f e ; p o ;\left[(W \cup R)^{s c}\right] \subseteq$

$\left[E^{s c}\right] ; p o_{\neq l o c} ; h b^{?} ; h b ; p o_{\neq l o c} ;\left[E^{s c}\right] \subseteq\left[E^{s c}\right] ; s c b ;\left[E^{s c}\right]$.

- Покажем, что $\left[E^{s c}\right] ; p o_{\neq l o c} ; h b^{?} ; C 2 ; r f e ; p o ;\left[(W \cup R)^{s c}\right] \subseteq\left(\left[E^{s c}\right] ; s c b ;\left[E^{s c}\right]\right)^{*}$. Заметим, что последовательность пар рёбер $r f e ; r m w$ входит в $s c b$ :

$C 2=\left[F^{a c q}\right] ; p o ;\left[E^{s c}\right] ;(\text { rfe } ; r m w)^{*}$

$\subseteq p o_{\neq l o c} ;\left[E^{s c}\right] ;\left(\left[E^{s c}\right] ; \text { rfe; }\left[E^{s c}\right] ; r m w ;\left[E^{s c}\right]\right)^{*} ;\left[E^{s c}\right]$

$\subseteq p o_{\neq l o c} ;\left[E^{s c}\right] ;\left(\left[E^{s c}\right] ; s c b ;\left[E^{s c}\right]\right)^{*} ;\left[E^{s c}\right]$.

В этом случае

$\left[E^{s c}\right] ; p o_{\neq l o c} ; h b^{?} ; C 2 ; r f e ; p o ;\left[(W \cup R)^{s c}\right]$

$\subseteq\left[E^{s c}\right] ; p o_{\neq l o c} ; h b^{?} ; p o_{\neq l o c}$. Тогда

$\left[E^{s c}\right] ;\left(\left[E^{s c}\right] ; s c b ;\left[E^{s c}\right]\right)^{*} ;\left[E^{s c}\right] ;$ rfe $\boldsymbol{p} ; p o ;\left[(W \cup R)^{s c}\right] \subseteq$

$\left(\left[E^{s c}\right] ; s c b ;\left[E^{s c}\right]\right) ;\left(\left[E^{s c}\right] ; s c b ;\left[E^{s c}\right]\right)^{*} ;\left(\left[E^{s c}\right] ; s c b ;\left[E^{s c}\right]\right)^{2}$. $\square$

\section{7. Связанные работь}

Проблема корректности схем компиляции из ОCamlMM и в IMM рассматривается и в других работах. Так, в [15] приводится схема компиляции ОСаmIMM в модель архитектуры ARMv8 [11]. В ней, в отличие от предложенной нами схемы, при компиляции неатомарной записи используется барьер $F^{a c q}$, а не $F^{\text {acqrel }}$. Это объясняется тем, что в модели ARMv8 отношение $o b$ (аналог $a r$ в IMM) включает в себя $r f e$ U fre U

coe по неатомарным операциям и ро с $a c q$-барьером перед событием записи, поэтому в последовательности рёбер вида $(p o ; r f e)^{+}$не требуется $r e l$-барьер.

B [17] приведена схема компиляции моделей RC11 [18] в IMM. Так как предикат согласованности IMM схож с таковым в RC11, то эта схема компиляции лишь незначительно отличается от тривиальной.

B [20], [21] и [22] разработана схема компиляции модели Promising [23] в модель ARMv8 [11].

Доказательство корректности данной схемы значительно сложнее, так как модель Promising, в отличие от модели ARMv8, задана с помощью операционной семантики, что требует при доказательстве корректности компиляции задавать соответствие между графами исполнения и последовательностями шагов операционной семантики. В [17] идея этого доказательства была обобщена для построения корректной схемы компиляции IMM B ARMv8. 


\section{Заключение}

В данной работе представлена корректная схема компиляции ОСamlMM в IMM, дающая корректную схему компиляции OCamlMM в модель Power. Для доказательства корректности было доказано, что в графах исполнения скомпилированных программ IMMсогласованность влечёт ОСam1MM-согласованность. Так как IMM является более слабой моделью, чем OCamlMM, в предложенной схеме компиляции задействуются барьеры памяти и инструкции compare-and-swap, которые накладывают более строгие условия на поведение скомпилированной программы.

Свойство локальной свободы от гонок может быть реализовано и в других моделях памяти- например, изучается возможность включить его в модель памяти С++ [24]. Данная работа может быть использована для построения схем компиляции таких моделей. В некоторых опубликованных доказательствах корректности компиляции впоследствии были найдены неточности. Например, [18] демонстрирует ошибку в схеме компиляции модели C++ в модель Power, [17] - в схеме компиляции модели Promising в модель Power. Чтобы избежать этого, доказательство в данной статье в дальнейшем планируется формализовать в Соq с использованием имеющейся формальной модели IMM [25].

\section{Список литературы / References}

1]. Lamport L. How to make a multiprocessor computer that correctly executes multiprocess programs. IEEE Transactions on Computers, vol. C-28, isssue 9, 1979, pp. 690-691.

2]. Owens S., Sarkar S., and Sewell P. A better x86 memory model: x86-TSO. Lecture Notes in Computer Science, vol. 5674, 2009, pp. 391-407.

[3]. Alglave J., Maranget L., Sarkar S., and Sewell P. Litmus: Running Tests Against Hardware. Lecture Notes in Computer Science, vol. 6605, 2011, pp. 41-44.

[4]. Batty M., Owens S., Sarkar S., Sewell P., and Weber T. Mathematizing C++ concurrency. In Proc. of the 38th annual ACM SIGPLAN-SIGACT symposium on Principles of programming languages, 2011, pp. 55-66.

[5]. Manson J., Pugh W., and Adve S.V. The Java memory model. In Proc. of the 32nd ACM SIGPLANSIGACT symposium on Principles of programming languages, 2005, pp. 378-391.

[6]. ECMA International. 2018b. ECMAScript 2018 Language Specification - Memory Model. Available at: https://www.ecma-international.

[7]. Watt C., Rossberg A., Pichon-Pharabod J. Weakening WebAssembly. Proceedings of the ACM on Programming Languages, vol. 3, issue OOPSLA, 2019, $28 \mathrm{p}$

8]. Sewell P., Sarkar S., Owens S., Nardelli F.Z., and Myreen M. O. x86-TSO: a rigorous and usable programmer's model for x86 multiprocessors. Communications of the. ACM, vol. 53, issue 7, 2010, pp. 89-97.

[9]. Alglave J., Maranget L., and Tautschnig M. Herding cats: Modelling, simulation, testing, and data mining for weak memory. ACM Transactions on Programming Languages and Systems, vol, 36, issue 2, 2014, pp. 7:1-7:74.

[10]. Sarkar S., Sewell P., Alglave J., Maranget L., and Williams D. Understanding POWER Multiprocessors. In Proceedings of the 32nd ACM SIGPLAN Conference on Programming Language Design and Implementation, 2011, pp. 175-186.

[11]. Pulte C., Flur S., Deacon W., French J., Sarkar S., and Sewell P. Simplifying ARM concurrency: Multicopy-atomic axiomatic and operational models for armv8. Proceedings of the ACM on Programming Languages, vol. 2, issue POPL, 2017, pp. 19:1-19:29.

[12]. ARM Limited. ARM architecture reference manual: ARMv7-A and ARMv7-R edition, 2014 Available

https://static.docs.arm.com/ddi0406/c/DDI0406C_C_arm_architecture_reference_manual.pdf.

[13]. Flur S., Gray K., Pulte C., Sarkar S., Sezgin A., Maranget L, Deacon W., and Sewell P. Modelling the ARMv8 architecture, operationally: Concurrency and ISA. In Proc. of the 43rd Annual ACM SIGPLAN-SIGACT Symposium on Principles of Programming Languages, 2016, pp. 608-621.

[14]. Dolan S., Sivaramakrishnan K., and Madhavapeddy A. Bounding data races in space and time. Extended version. Available at: http://kcsrk.info/papers/pldi18-memory.pdf.
[15]. Dolan S., Sivaramakrishnan K., and Madhavapeddy A. Bounding data races in space and time. In Proc. of the 39th ACM SIGPLAN Conference on Programming Language Design and Implementation, of the 39th ACM

[16]. IBM Power Systems. IBM power systems facts and features: enterprise and scale-out systems with POWER8 processor technology. Available at: https://www.ibm.com/downloads/cas/JDRZDG0A.

[17]. Podkopaev A., Lahav O., and Vafeiadis V. Bridging the gap between programming languages and hardware weak memory models. Proceedings of the ACM on Programming Languages, vol. 3, issue POPL, 2019, pp. 69:1-69:31.

[18]. Lahav O., Vafeiadis V., Kang J., Hur C.-K., and Dreyer D. Repairing sequential consistency in $\mathrm{C} / \mathrm{C}++11$. Programming Language Design and Implementation, vol. 52, issue 6, 2017, pp. 618-632.

[19]. Podkopaev A., Lahav O., Melkonian O., and Vafeiadis V. Extending Intermediate Memory Mode with SC accesses. Technical report. Available at: http://plv.mpi-sws.org/imm/immsctr.pdf. 2019

[20]. Подкопаев А.В. Операционные методы в приложениях к слабым моделям памяти. Диссерташия на соискание учёной степени кандидата физико-математических наук. Санкт-Петербург, 2018 190 стр. / Podkopaev A.V. Operational methods in applications to weak memory models. The dissertation for the degree of candidate of physical and mathematical sciences. St. Petersburg, 2018,190 p. (in Russian).

[21]. Подкопаев А.В., Лахав О., Вафеядис В. О корректности компиляции подмножества обещающей модели памяти в аксиоматическую модель ARMv8.3. Научно-технические обещающей модели памяти в аксиоматическую модель ARMv8.3. Научно-технические

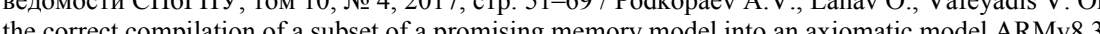
the correct compilation of a subset of a promising menory model into an axionatic model ARMv8.3. St. Petersburg Polytechnic University Journal of Engineering Science and Technology, vol. 10, № 4 pp. 51-69 (in Russian).

22]. Подкопаев А.В., Лахав О., Вафеядис В. Обещающая компиляция в ARMv8.3. Труды ИСП РАН, том 29, вып. 5, 2017, стр. 149-164 / Podkopaev A.V., Lahav O., Vafeiadis V. Promising Compilation to ARMv8.3. Trudy ISP RAN/Proc. ISP RAS, vol. 29, issue 5, 2017. pp. 149-164 (in Russian). DOI 10.15514/ISPRAS-2017-29(5)-9.

[23]. Kang J., Hur C.-K., Lahav O., Vafeiadis V., and Dreyer D. A promising semantics for relaxed-memory concurrency. In Proc. of the 44th ACM SIGPLAN Symposium on Principles of Programming Languages, 2017, pp. 175-189.

[24]. Doherty S. Local data-race freedom and the C11 memory model. Surrey Concurrency Workshop Absracts, 2019. Available at: https://cw-srepls-19.github.io/abstracts.html\#doherty/

[25]. Coq formalization of the Intermediate Memory Model. Available at: https://github.com/weakmemory/imm. 2019.

\section{Информация об авторах / Information about authors}

Егор Сергеевич НАМАКОНОВ - студент магистратуры Санкт-Петербургского государственного университета, член группы исследования слабых моделей памяти в JetBrains Research. Сферы научных интересов: слабые модели памяти, верификация ПО.

Egor Sergeevich NAMAKONOV - a Master's student in St Petersburg University, a member of weak memory model research group in JetBrains Research. Research interests: weak memory models, software verification.

Антон Викторович ПОДКОПАЕВ - доцент НИУ ВШЭ (СПб), руководитель группь исследования слабых моделей памяти в JetBrains Research, постдок Институт им. Макса Планка: Программные Системы. Сферы научных интересов: слабые модели памяти, верификация ПО, функциональное программирование.

Anton Viktorovich PODKOPAEV - an associate professor in NRU HSE (SPb), the weak memory model group leader in JetBrains Research, a postdoc in MPI-SWS. Research interests: weak memory models, software verification, functional programming. 